\title{
Efectos de un gel de tetraciclina al $5 \%$ sobre los niveles de P. gingivalis, P. intermedia y A. actinomycetemcomitans
}

\author{
GALIARDO $\mathbf{F}$ * \\ PLAZA JC ** \\ DE LA SOTTA $\mathbf{R} * * *$
}

\begin{abstract}
Gallardo F, Plaza JC, De la Sotta R. Efectos de un gel de tetraciclina al 5\% sobre los niveles de P. gingivalis, P. intermedia y A. actinomycetemcomitans. Av Periodon Implantol. 2002; 14, 1:21-28.
\end{abstract}

\begin{abstract}
RESUMEN
El objetivo de la presente investigación fue estudiar los efectos de un gel de tetraciclina al $5 \%$ sobre los niveles de 3 microorganismos asociados al desarrollo de la periodontitis rápidamente progresiva (PRP). En un total de 20 pacientes con PRP se seleccionaron 5 dientes por paciente con bolsas periodontales $\geq$ a $5 \mathrm{~mm}$. con sangrado al sondaje periodontal en los cuales se efectuaron los siguientes tratamientos: 1 . Control. 2. Aplicación de un gel de tetraciclina al 5\% (T). 3. Placebo (Pl). 4. Raspado y alisado radicular (RA). 5. T + RA .De forma previa, en cada sitio seleccionado, se tomaron muestras de placa subgingival con un cono de papel estéril para detectar y cuantificar la presencia de $P$. gingivalis , $P$. intermedia y $A$. actinomycetemcomitans, mediante el uso de sondas DNA (OMNIGENE, U.S.A.). Se dieron instrucciones de higiene oral, y se efectuó un nuevo control microbiológico a los 60 días

El análisis estadístico de los resultados demostró lo siguiente: 1 . Ninguno de los tratamientos redujo significativamente los niveles de $A$. actinomycetemcomitans. 2 . Se detectó una reducción significativa de $P$. gingivalis en los sitios tratados con (R.A). $(\mathrm{p}<0.02)$ o cuando este ultimo tratamiento se asoció a $(T) .(p<0.05) 3$. Todos los tratamientos redujeron significativamente los niveles de $P$. intermedia.

Aunque las tetraciclinas incluyen en su espectro de acción a las bacterias estudiadas, y alcanzan altas concentración después de su aplicación en los sitios respectivos, los reducidos efectos microbiológicos encontrados podrían explicarse por su rápida remoción por el fluido gingival desde su sitio de aplicación en las localizaciones estudiadas.
\end{abstract}

\section{PALABRAS CLAVE}

Tetraciclina gel - Aplicación Local - Bacterias periodontales - Periodontitis rápidamente porogresiva

\section{INTRODUCCIÓN}

Las enfermedades periodontales, gingivitis y periodontitis, comprometen los tejidos del periodoncio, presentando una elevada prevalencia en nuestra población. El tratamiento de estas afecciones es un importante problema de salud pública porque su omisión deriva en la pérdida de piezas dentarias, lo que trae consecuencias estéticas, funcionales y psicológi-

* Profesor Asociado de Farmacología, Instituto de Ciencias Biomédicas, Programa de Farmacología Molecular y Clínica, Facultad de Medicina, Universidad de Chile

** Profesor Asociado de Periodoncia. Escuela de Graduados, Sección Periodoncia, Facultad de Odontología, Universidad de Chile.

*** Profesor Titular de Periodoncia. Escuela de Graduados, Sección Periodoncia, Facultad de Odontología, Universidad de Chile. 


\section{AVANCES}

Volumen 14 - N$^{\circ} 1$ - Abril 2002

cas en el paciente. Adicionalmente, evidencias disponibles en la última década señalan que la periodontitis es un factor de riesgo para el desarrollo de diversas patologías cardiovasculares, respiratorias, obstétricas etc. (1-4). En atención a lo anterior el tratamiento de las enfermedades periodontales es de la mayor importancia no sólo por la salud de los tejidos del periodonto sino también para preservar la salud general del individuo.

Es indudable que el factor etiológico de mayor relevancia para el desarrollo de las enfermedades periodontales es la flora microbiana, y para la gran mayoría de los investigadores, las enfermedades periodontales corresponden a infecciones específicas producidas por una proliferación de algunas de las 300 o más especies microbianas que se han detectado en el ecosistema subgingival. En efecto, las investigaciones efectuadas con técnicas microbiológicas, inmunológicas y mediante el empleo de sondas que detectan el DNA bacteriano indican con escasas dudas que la flora asociada al desarrollo de las periodonttis incluye bacterias anaerobias estrictas, como Porphyromonas gingivalis, Prevotella intermedia, Bacteroides forsythus, Treponema denticola, Campylobacter rectus, Fusobacterium nucleatum, Peptostreptococcus micros, aunque también se ha detectado la presencia de especies facultativas como por ejemplo Actinobacillus actinomycetemcomitans y Eikenella corrodens.(5-10)

Dado que la flora microbiana juega un rol etiológico fundamental para el desarrollo de las diversas formas de periodontitis, se ha propuesto que el uso sistémico de algunos antimicrobianos como tetraciclinas, metronidazol, amoxicilina etc. constituiría un adecuado complemento a la terapia mecánica inespecífica convencional (raspado y pulido radicular), para permitir una mejor eliminación de las bacterias que constituyen el factor etiológico fundamental (11-23). Aquellas investigaciones, efectuadas con diferentes diseños, han demostrado que el uso sistémico de los antimicrobianos mencionados es un útil complemento a la terapia mecánica convencional, especialmente en aquellas formas de periodontitis más agresivas como las denominadas periodontitis de inicio precoz, que incluyen las formas prepuberal, juvenil y la rápidamente progresiva y a las denominadas formas refractarias de periodontitis

Sin embargo una terapia sistémica no está exenta de producir reacciones adversas, de mayor o menor grado dependiendo del antibiótico utilizado, al exponer todo el organismo durante un período que oscila entre una semana y 3 semanas a los efectos del antibiótico seleccionado, lo que ha concitado el interés de los investigadores por el uso local de antimicrobianos, aplicados profesionalmente en las bolsas periodontales, entre los cuales cabe mencionar a tetraciclinas, clorhexidina, metronidazol etc., disponibles mediante diversos vehículos en los sitios respectivos (soluciones, geles, fibras, comprimidos de liberación lenta etc.).

El objetivo de la presente investigación consistió en determinar la eficacia de la aplicación local de un gel de tetraciclina al 5\% en bolsas profundas de pacientes con el diagnóstico clínico radiográfico de periodontitis rápidamente progresiva. Los resultados que se presentan a continuación corresponden a los efectos de las terapias antibiótica y/o mecánica sobre los niveles de 3 periodontopatógenos importantes en el desarrollo estas afecciones, P.gingivalis, P.intermedia y A.Actinomycetemcomitans, y una futura comunicación incluirá los efectos de los tratamientos sobre diferentes parámetros clínicos de la enfermedad.

\section{MATERIAL Y MÉTODO}

La investigación se efectuó en un grupo de pacientes que fueron atendidos en la Clínica de Periodoncia de la Escuela de Graduados, Universidad de Chile, con el diagnóstico de periodontitis rápidamente progresiva, de acuerdo con la descripción de Page y col. (24 ) La muestra estuvo conformada por 20 pacientes, 17 mujeres y 3 hombres entre 28 a 40 años con una edad promedio de $32.7 \pm 1.53$ años.

Después que los pacientes aceptaron ser incluídos en el ensayo clínico, se incorporaron al estudio siempre que no hubieran recibido antibióticos ni colutorios bucales en los 6 meses precedentes al inicio del estudio, no fueran diabéticos ni tuvieran antecedentes de hipersensibilidad a las tetraciclinas. Asimismo no se incluyeron las pacientes embarazadas ni aquellas en etapa de lactancia.

Posterior al diagnóstico de la afección, se confeccionó una ficha ad-hoc para cada paciente que incluyó diversos parámetros clínicos cuyos resultados se reportarán con posterioridad. A continuación se efectuó un detartraje supragingival en ambos maxilares mediante ultrasonido e instrumentación mecánica y se procedió a seleccionar en cada paciente 5 dientes no adyacentes, superiores o inferiores que presentaran bolsas de una profundidad $\geq$ a $5 \mathrm{~mm}$. asociados a sangrado al sondaje periodontal y evidencias radiográficas de reabsorción alveolar.

En cada diente seleccionado se escogió el sitio más profundo y después de aislar el campo operatorio, se tomó una muestra de placa subgingival mediante un cono de papel estéril, el cual se mantuvo 10 segundos en el sitio y luego se depositó en un tubo Eppendorf de plástico. Posteriormente, se identificó el tubo respectivo, se completó un formulario anexo y las 5 
muestras de cada paciente se depositaron en una bolsa de plástico y se envió por Correo aéreo rápido a OMNIGENE, Cambridge, Massachussets U.S.A., donde cada muestra se procesó mediante sondas DNA para identificar y cuantificar la presencia de los periodontopatógenos, $P$ gingivalis, $P$ intermedia y $A$. Actinomycetemcomitans.

En una nueva sesión y en cada una de los 5 dientes seleccionados se efectuó uno de los siguientes tratamientos:

1. Aplicación local de tetraciclina gel al 5\% (elaborada por Laboratorio Chile S.A.), mediante el uso de Periopik (Waterpik Teledyne Co., U.S.A.). Después de aislar el campo operatorio con rollos de algodón, se procedió a cargar el dispositivo respectivo con el antibiótico y se depositó su contenido en el sitio seleccionado durante un período de 3 minutos, con la precaución que los pacientes no se enjuagaran después de dicha maniobra.

2. Aplicación local de un placebo. Utilizando una técnica similar a la descrita anteriormente, se procedió a depositar un gel-placebo, que tenía el mismo aspecto del compuesto activo, con el fin de preservar la naturaleza doble-ciego de la investigación.

3. Raspaje y alisado radicular, mediante curetas Gracey, el cual se efectuó en una hemiarcada, dejando la opuesta como control.

4. Raspaje y alisado radicular asociado a la aplicación local del gel activo.

5. Control, es decir, éste último diente seleccionado no recibió terapia antibiótica ni mecánica.

A continuación, se instruyó a cada paciente para efectuar una óptima higiene oral mediante una técnica de cepillado y uso de seda dental; se citó a control clínico a los 30 y 60 días, se efectuó una nueva toma de muestra de placa subgingival para el análisis microbiológico sólo a los 60 días.

Cabe destacar que el estudio se efectuó utilizando el método doble-ciego, de manera que ni el operador ni el paciente conocieron la naturaleza de las medicaciones utilizadas, las que sólo fueron identificadas mediante un código conocido sólo por un investigador que no participó en el control clínico de los pacientes.

\section{Análisis estadístico}

Se calcularon en forma separada, los valores medios \pm error típico de cada una de las 3 bacterias estudiadas en los sitios seleccionados al comienzo (día 0) y a los
60 días, los cuales se compararon estadísticamente mediante el test " $t$ " de Student para muestras pareadas, considerando significativos todos aquellos resultados que tuvieran un valor $\mathrm{p}<0.05$.

\section{RESULTADOS}

La prevalencia inicial de cada bacteria en los 100 sitios estudiados fue reportada anteriormente (25). Se demostró que A.actinomycetemcomitans estaba presente sólo en 9 del total de 100 muestras analizadas y en niveles bajos, mientras que se detectaron $P$. gingivalis y $P$. intermedia en la mayoría de los sitios y en niveles altos (superiores a 600.000 células). Asimismo, en la investigación mencionada se demostró una correlación positiva entre la presencia de P. gingivalis y los parámetros clínicos profundidad de las bolsas y nivel de inserción ( $\mathrm{p}<0.01$ y 0.02 respectivamente) y también hubo una correlación positiva entre $P$. Intermedia y el parámetro profundidad de las bolsas $(p<0.03)$.

\section{Efectos sobre los niveles de A. actinomycetemcomitans}

En la Fig. 1 aparecen los efectos de los tratamientos sobre A.actinomycetemcomitans. Como puede apreciarse, no se detectó una reducción significativa en los

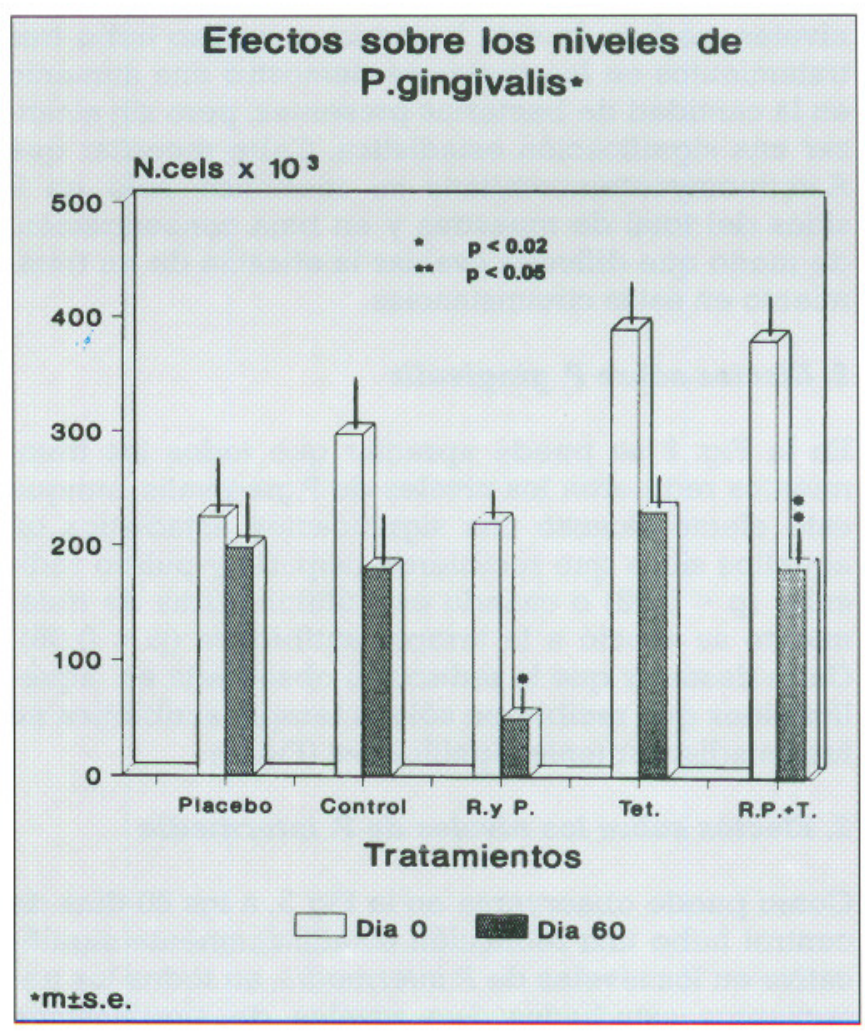

Fig. 1 


\section{AVANCES}

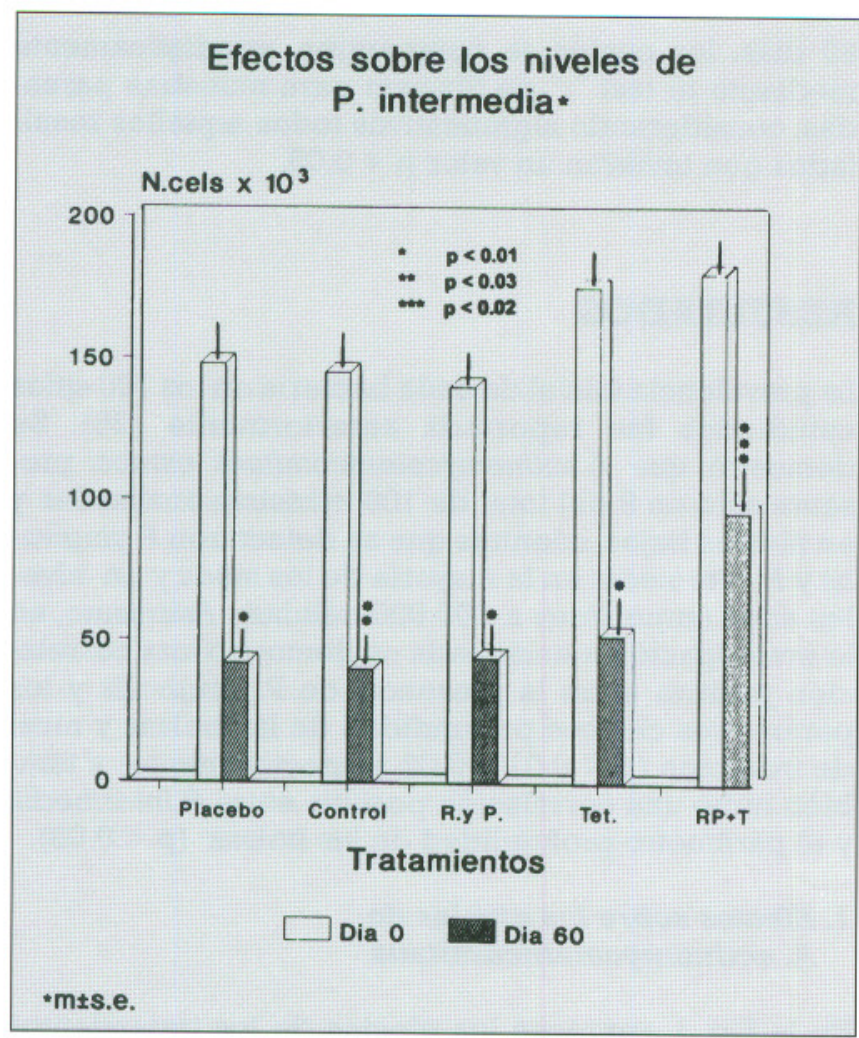

Fig. 2

niveles medios de esta bacteria, e incluso hubo tres tratamientos en los cuales se demostró una aumento en la cantidad de bacterias presentes, pero sin alcanzar una significación estadística. Cabe recordar que A.actinomycetemcomitans se identificó solo en 9 sitios del total de muestras y en baja concentración, de modo que dificulta evaluar la eficacia de un tratamiento en estas circunstancias.

\section{Efectos sobre P. gingivalis}

En la Fig. 2 se puede apreciar que todos los tratamientos redujeron los niveles de P.gingivalis, aunque este efecto alcanzó una significación estadística en aquellos sitios que recibieron raspado y pulido radicular $(\mathrm{p}<0,02)$ o cuando esta última forma de tratamiento se asoció a la terapia antibiótica $(p<0.05)$. Cabe destacar que la reducción observada en aquellos sitios que recibieron sólo la terapia antibiótica no fue estadísticamente significativa (Fig. 2).

\section{Efectos sobre los niveles de P. intermedia}

Como puede observarse en la Fig 3, a los 60 días de control hubo una reducción estadísticamente significativa en los niveles de $P$. intermedia, en todos los tratamientos estudiados. Los niveles de significación aparecen en la Fig. 3

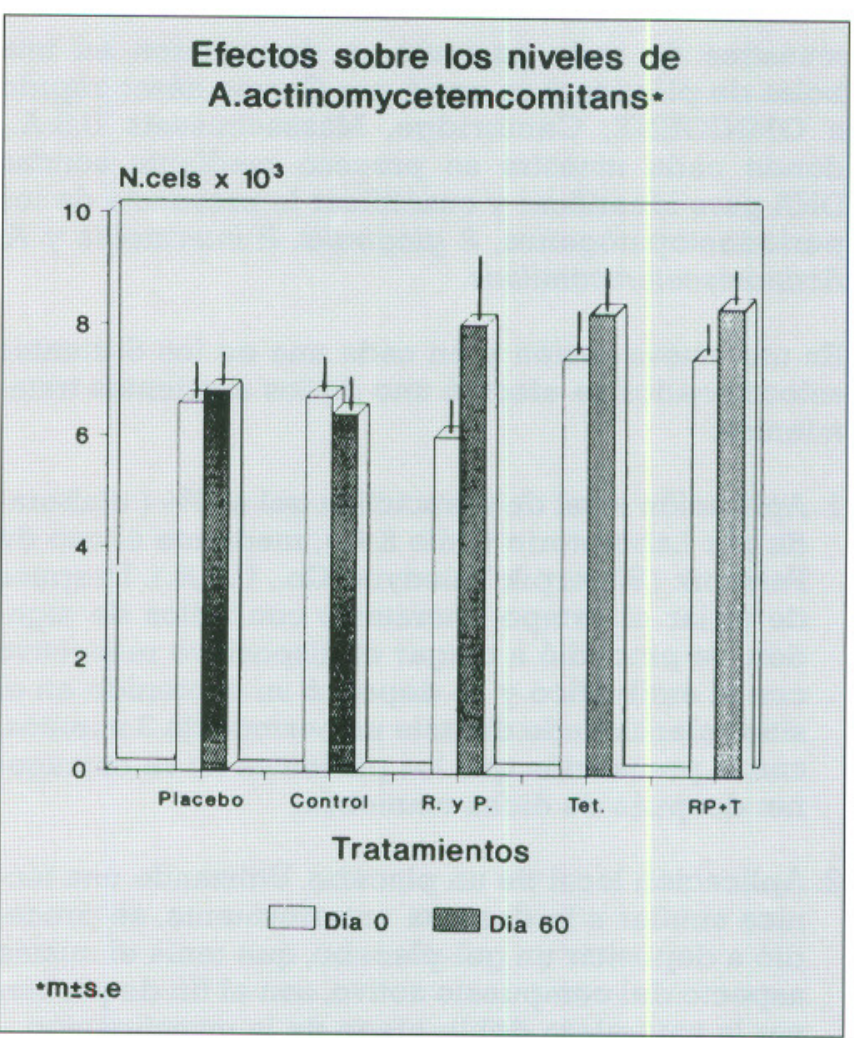

Fig. 3

\section{DISCUSIÓN}

El diagnóstico de las enfermedades periodontales, particularmente de las diversas formas de periodontitis ha experimentado un considerable progreso con la incorporación de la técnica de las sondas DNA, un aporte de la biología molecular que identifica secuencias específicas de nucleótidos en la estructura del DNA microbiano. A causa de lo anterior, la técnica tiene alta sensibilidad y especificidad, con un límite de detección de 6.000 células, una cantidad bastante reducida si consideramos que la técnica de cultivo tradicional requiere al menos la presencia de 100.000 células.(26-29). Además de lo anterior, la técnica no requiere la presencia de células viables, porque procesa el DNA bacteriano, lo que favorece la toma de muestras y su transporte hacia el laboratorio respectivo.

Utilizando la técnica mencionada, en la presente investigación se demostró que una sola aplicación local de un gel de tetraciclina al $5 \%$ en pacientes con periodontitis rápidamente progresiva redujo significativamente los niveles de $P$. intermedia aunque estos efectos también aparecieron en aquellos sitios no tratados o que recibieron un placebo y fueron similares a aquellos producidos por el raspaje y alisado radicular, lo que demuestra el escaso efecto del antibiótico 
frente a esta bacteria. Adicionalmente, el antibiótico como única medida terapéutica no redujo significativamente los niveles de $P$. gingivalis, un efecto que sí apareció en aquellos sitios que recibieron la terapia mecánica convencional, lo que también confirma la reducida eficacia del antibiótico frente a esta bacteria. Por último, tampoco la terapia antibiótica determinó una reducción significativa sobre $A$. actinomycetemcomitans, aunque el número de sitios positivos para esta bacteria el día 0 fue bastante reducido, lo que dificulta la evaluación de la terapia antibiótica local utilizada.

En atención a las características farmacológicas de las tetraciclinas, que destacan por su actividad frente a la flora estudiada (30-32), por las altas concentraciones obtenidas en los tejidos del periodonto siguiente a su administración sistémica $(33,34)$ y a la sustantividad del medicamento en los sitios estudiados (35), llama la atención los reducidos efectos sobre las bacterias estudiadas, de manera que es necesario mencionar las posibles causas de los limitados efectos encontrados.

En primer término, es preciso recordar que los pacientes recibieron una sola aplicación del antibiótico al comienzo del estudio, lo que podría ser insuficiente. En efecto, Van Steenberghe y col. (36) demostraron significativas reducciones de A.actinomycetemcomitans. $P$. gingivalis y $P$. intermedia con el uso de un gel de minociclina al $2 \%$ a las 2, 4,6 y 12 semanas de control, pero en el estudio mencionado el antibiótico se depositó en 4 oportunidades, al comienzo y a los 2, 4 y 6 semanas. Por otra parte, el uso de un gel de tetraciclina al $40 \%$, (37) redujo significativamente los porcentajes de espiroquetas y bacilos móviles y aumentó las formas cocáceas a las 8 y 12 semanas de tratamiento, lo que demuestra la utilidad de una mayor concentración del antibiótico.

Empleando una mayor frecuencia de aplicación de un antibiótico, otros estudios con una solución de tetraciclina al 5\%, han demostrado una interesante actividad antimicrobiana sobre la flora subgingival (38), mientras que Mac Alpine y col. (39) al utilizar una irrigación con una solución de tetraciclina al 5\%, 2 veces por semana durante 24 semanas en pacientes con bolsas profundas, no pudieron demostrar un efecto aditivo del antibiótico a la terapia mecánica convencional, aunque el número de sitios estudiados fue limitado.

Probablemente el principal factor responsable de la reducida actividad antibiótica local es la remoción del producto desde su sitio de aplicación. En efecto, el constante flujo del fluído gingival, que experimenta un proceso de recambio varias veces durante una hora (40), es capaz de remover con facilidad un medi- camento depositado en forma de una solución en el medio subgingival, dado que se ha estimado que la vida media del medicamento en este sitio (el tiempo que transcurre hasta que su concentración decrece en un $50 \%$ ) es aproximadamente un minuto (40). Indudablemente que esta rápida eliminación de un medicamento depositado en el surco gingival impide una acción sostenida sobre las bacterias de la placa subgingival, lo que redunda en una acción antimicrobiana insuficiente sobre aquellas. Si bien es cierto que en esta investigación el medicamento se incluyó en un vehículo diferente (gel) del cual cabría esperar una eliminación más lenta desde su sitio de aplicación, como no se midieron las concentraciones del antibiótico al comienzo y en días posteriores, el limitado efecto antimicrobiano encontrado puede explicarse por su rápida eliminación desde los sitios respectivos.

Por último, cabe consignar que una alta concentración de un antibiótico en el surco gingival no necesariamente puede asociarse a un marcado efecto antimicrobiano, porque la flora periodontal se organiza formando una estructura compleja que se ha denominado biofilm la cual dificulta la difusión o inactiva un antibiótico (41-43). Por consiguiente, el efecto de un antimicrobiano sobre bacterias dispuestas en un biofilm puede requerir concentraciones mayores que aquellas detectadas in vitro, cuando estas se encuentran en un medio convencional de cultivo (41-43).

En consideración a las limitaciones señaladas, los investigadores han diseñado sistemas de liberación sostenida de antimicrobianos en el surco gingival, entre los que destacan las fibras de etilenvinilacetato impregnadas en tetraciclina al 25\% $(44,45)$, estructuras sólidas biodegradables que contienen clorhexidina $(46,47)$, que persisten alrededor de una semana en el surco gingival o un gel que contiene metronidazol $(48,49)$ o un polímero degradable que incluye doxiciclina hiclato,(50,51) los cuales liberan el antibiótico por un período prolongado. Los sistemas mencionados representan mecanismos de liberación sostenida y controlada en el surco gingival, lo que explica los positivos resultados que se han obtenido en los últimos años con estos productos.

\section{AGRADECIMIENTOS}

Los autores expresan su sincero agradecimiento a Laboratorio Chile, Santiago Chile, quienes elaboraron el antibiótico y el placebo utilizado en la investigación. También agradecen a Waterpik Teledyne Co., quiénes financiaron parcialmente el estudio microbiológico efectuado. 


\section{SUMMARY}

The purpose of this investigation was to study the effects of local delivery of a tetracycline $5 \%$ gel on the levels of 3 bacteria associated to development of rapidly progressive periodontitis. In a sample of 20 patients, five teeth were selected from each patient with periodontal pockets $\geq 5 \mathrm{~mm}$ and bleeding upon probing. One of the following treatments were done at each selected site: 1 . Control (no treatment). 2. Local delivery of a $5 \%$ tetracycline gel. 3. Placebo gel. 4 . Scaling and root planing. 5. Scaling and root planing + local application of tetracycline gel. Previously, at each selected site samples of subgingival plaque were taken with sterile paper points in order to detecte and quantify the presence of $P$. gingivalis, $P$. intermedia and $A$. actinomycetemcomitans, by using DNA probe technology (OMNIGENE, U.S.A.).

Oral hygiene instructions were given to each patient and new samples of subgingival plaque were obtained at 60 days.

Statistical analysis of results showed the following 1 . No significant reductions of $A$. actinomycetemcomitans were found with performed treatments. 2. Scaling and root planing reduced the levels of $P$. gingivalis $(\mathrm{p}<$ 0.02 ) or when the later was combined with tetracycline $(p<0.05)$. 3. All treatments significantly reduced levels of P.intermedia.

Although bacteria studied has shown sensitivity to tetracyclines and despite high levels of antibiotic that are obtained following its local delivery in a gel, the reduced microbiologic effects that were seen could be ascribed to rapid removal of gel by the gingival crevicular fluid from studied sites

\section{KEY WORDS}

Tetracycline gel - Local delivery - Effects on periodontal bacteria - rapidly progressive periodontitis.

\section{CORRESPONDENCIA}

Dr. Fernando Gallardo R

Casilla 70.000 Correo - 7 Santiago. Chile

e- mail : fgallard@machi.med.uchile.cl

\section{BIBLIOGRAFÍA}

1. Mattila K.J., Valtonen V.V., Nieminem M,Huttunen J.K.
Dental infection and the risk of new coronary events: prospective study of patients with documented coronary artery disease. Clin Infect Dis 1995; 20: 588-592

2. Beck J.D., García R.J., Heiss G., Vokonas P.S., Offenbacher S. Periodontal disease and cardiovascular disease. J.Periodontol 1996; 67:1123-1137

3. Scannapieco F.A., Stewart E.M.,Mylotte J.M. Colonization of dental plaque by respiratory pathogens in medical intensive care patients. Crit Care Med 1992; 20:740-745

4. Collins J.G.,Windley H.W.,Arnold R.R., Offenbacher S. Effects of Porphyromonas gingivalis infection on inflammatory mediator response and pregnancy outcome in hamsters. Infect Immun 1994; 32: 4356-4361

5. Socransky S.S. Microbiology of periodontal disease Present status and future considerations. J.Periodontol 1977,48:497-504

6. Slots J. Subgingival microflora and periodontal disease. J.Clin.Periodontol. 1979; 6:351-382,

7. Slots J., Listgarten M.A. Bacteroides gingivalis, Bacteroides intermedius and Actinobacillus actinomycetemcomi-tans in human periodontal diseases. J.Clin. Periodontol. 1988; 15: 85-93

8. Slots J. Bacterial specificity in adult periodontitis. Summary of recent work. J. Clin. Periodontol. 1986; 13:912-917

9. Loesche W.J., Syed S.A., Schmidt E., Morrison E.C. Bacterial profiles of subgingival plaques in periodontitis. J.Periodontol. 1985; 56,447-456

10. Socransky SS.,Haffajee AD. The bacterial etiology of destructive periodontal disease: Current concepts. J.Periodontol 1992; 63:322-331,

11. Loesche W.J. The antimicrobial treatment of periodontal disease Changing the treatment paradigm. Crit. Rev Oral Biol Med 1999; 10: 245-275,

12. Loesche W.J., Syed S.A., Morrison E.C., Kerry G.A et al. Metronidazole in periodontitis 1 . Clinical and bacteriologic results after 15 tom 30 weeks. J.Periodontol 1984; 55 : 325-335

13. Lindhe J., Liljenberg B. Treatment of localized juvenile periodontitis. Results after 5 years. J.Clin Periodontol $1984 ; 11: 399-410$

14. Van Winkelhoff A.J., Tijhof C.LJ., de Graaf J. Microbiological and clinical results of metronidazole plus amoxicillin therapy in Actinobacillus actinomycetemcomitans-associated periodontitis. J.Periodontol 1992; 63:52-57 
15. Aitken S., Birek P., Kulkarni G.V., McCulloch C.A. Serial doxycicline and metronidazole in prevention of recurrent periodontitis in high risk patients. J.Periodontol $1992 ; 63: 87-92$

16. Asikainen S.,Jousimies-Somer H., Kanervo A., Saxen L. The inmediate efficacy of adjunctive doxycycline in treatment of localized juvenile periodontitis. Arch Oral Biol 1990; 35 (Suppl) $231-234$

17. Christersson L.A., Zambon JJ. Supression of subgingival Actinobacillus actinomycetemcomitans in localized juvenile periodontitis by systemic tetracycline. J.Clin Periodontol 1993;20:395-401

18. Goodson J.M. Antimicrobial strategies for treatment of periodontal diseases. Periodontology 2000 1994; 5:142-168

19. Gordon J., Walker C., Hovliaras C., Socransky S.S. Efficacy of clindamycin hydrochloride in refractory periodontitis: 24 month results. J.Periodontol 1990; 61:686-691

20. Saxen L., Asikainen S. Metronidazole in the treatment of localized juvenile periodontitis. J.Clin Periodontol 993; 20:166-171

21. López N., Gamonal J.A. Effects of metronidazole plus amoxycillin in progressive untreated adult periodontitis: report of a single 1- week course after 2 and 4 month. J.Periodontol 1998; 69:1291-1298

22. Gallardo F., Huerta J., Rossi E., Cruz E. Efectos de la doxiciclina asociada al raspaje y alisado radicular en el tratamiento de la enfermedad periodontal del adulto. Resultados a los 30, 90 y 180 días. Av.Periodoncia 1993; 5: 91-102

23. Gallardo F., Huerta J., Cruz E.,et al. Efectos del tinidazol en el tratamiento de la enfermedad periodontal del adulto.Estudio clínico y microbiológico. Av. Periodon. Implant Oral 1994; 6:161-174

24. Page R.C., Altman L.C.,Ebersole J.L. Rapidly progressive periodontitis. A distinct clinical condition. J.Periodontol 1983; 54:197-209

25. Gallardo F., Plaza J.C., de la Sotta R. Prevalencia de Porphyromonas gingivalis, Prevotella intermedia y Actinobacillus actinomycetemcomitans en pacientes con periodontitis rápidamente progresiva. Medicina Oral 2000; 5:151-158

26. French C.K.,Savitt E.D. Peros W.J. et al. DNA probe detection of periodontal pathogens. Oral Microbiol Inmmunol $1986 ; 1: 58-62$

21. Savitt E.D.,Strzempko M.N.,Vaccaro K.K. et al. Comparison of cultural methods and DNA probe analy- ses for the detection of Actinobacillus actinomycetemcomitans, Bacteroides gingivalis, and Bacteroides intermedius in subgingival plaque samples. J.Periodontology 1988; 59:431-438

28. Kisby L.B., Savitt E.D., French C.K.,Peros W.J. DNA probe detection of key periodontal pathogens in juveniles. J.Pedodontics 1989; 13:222-229

29. Lippke J.A.,Peros W.J., Savitt E.D.,French C.K. DNA probe detection of Eikenella corrodens, Wollinella recta and Fusobacterium nucleatum in subgingival plaque. Oral Microbiol Inmmunol 1991; 6:81-87

30. Baker P.J., Evans R.T., Slots J., Genco R. Susceptibility of human oral anaerobic bacteria to antibiotics suitable for topical use. J.Clin Periodontol 1985; 12: $201-208$

31. Walker C.B., Pappas J.D.,Tyler K.Z., Cohen S., Gordon J.M. Antibiotic susceptibilities of periodontal bacteria. In vitro susceptibilities to eight antimicrobial agents. J. Peridontol Suppl 1985; 56: 67-74

32. O'Connor B.C., Newman H.N., Wilson M. Susceptibility and resistance of plaque bacteria to minocycline. J.Periodontol 1990; 61:228-233

33. Gordon J.M.,Walker C., Murphy J.C. et al. Concentration of tetracycline in human gingival fluid after single doses. J.Clin Periodontol 1981; 8:117-121

34. Pascale D., Gordon J., Lamster I., et al. Concentration of doxycycline in human gingival fluid. J.Clin Periodontol 1986; 13:841-844

35. Baker P.J., Evans R.T., Coburn R.A., Genco R.J. Tetracyclines and its derivatives strongly bind to and are released from the tooth surface in active form. J.Periodontol 1983; 54:580-585

36. Van Steenberghe D., Bercy P., Kohl J et al. Subgingival minocycline ointment in moderate to severe chronic adult periodontitis: A randomized double-blind vehicle controlled multicenter study. J.Periodontol 1993; 64:637-644

37. Eckles T.A., Reinhardt R.A., Dyer J.K., Tussing G.J., Szydlowski W.M.,DuBous L.M. Intracrevicular application of tetracycline in white petrolatum for the treatment of periodontal disease. J.Clin Periodontol 17:454-462, 1990

38. Alfant M., Walker C.B., Bhaskar P. Local delivery of tetracycline as a possible adjunct to conventional periodontal therapy. J.Dent Res 1983; 62:289, (abstr)

39. MacAlpine R., Magnusson I et al. Antimicrobial irrigation of deep pockets to supplement oral hygiene instructions and root debridement 1 . Bi-weekly irrigation. J.Clin Periodoontol 1985; 12:568-577 
40. Goodson J. Pharmacokinetic principles controlling efficacy of oral therapy. J.Dent Res 1989; 68:1625-1632

41. Anwar H., Strap J. Costerton J. Establisment of aging biofilms: possible mechanism of bacterial resitance to antimicrobial therapy. Antimicrob Agents Chemother 1992; 36: 1347-1351

42. Cargill K., Pyle B., Sauer R. et al. Effects of culture conditions and biofilm formation on the iodine susceptibility of Legionella neumophila. Can J Microbiol 1992; 38: 423429

43. Brown M., Gilbert P. Sensitivity of biofilms to antimicrobial agents. J.Appl Bacteriol 1993; 74 ( Suppl) 87S - 97S

44. Goodson J.A., Tanner A., McArdle S. Dix K., Watanabe S. Multi-center evaluation of tetracycline fiber therapy 111. Microbiological response. J.Period Res 1991; 26:380-391

45. Lowenguth R.A., Caton J., Chin I. et al. Evaluation of periodontal treatments using controlled-release tetracycline fibers: microbiological response. J.Periodontol 1995; 66:700-707

46. Soskolne W., Heasman P., Stabholz A. et al. Sustained local delivery of chlorhexidine in the treatment of periodontitis: a multi-center study. J.Periodontol 1997; 68:32-38
47. Jeffcoat M, Bray K., Ciancio S. et al. Adjunctive use of a controlled release chlorhexidine chip reduces probing depths and improves attachment level compared with scaling and root planing alone. J.Periodontol 1998; 69: 989-997

48. Stelzel M., Flores de Jacoby L. Topical metronidazole application compared with subgingival scaling. A clini$\mathrm{cal}$ and microbiological study on recall patients. J. Clin Periodontol 1996; 23:24-29

49. Rudhart A., Purucker P., Kage A et al. Local metronidazole application in maintenance patients. Clinical and microbiological evaluation. J. Periodontol 1998; 69:11481154

50. Polson A., Garrett S., Stoller N et al. Multicenter comparative evaluation of subgingivally delivered sanguinarine and doxycycline in the treatment of periodontitis. J. Periodontol 1997; 68:119-126

51. Garret S, Johnson L., Drisko C.H. et al. Two multicenter studies evaluating locally delivered doxycycline hyclate, placebo control, oral hygiene and scaling and root planing in the treatment of periodontitis. J.Periodontol 1999; 70:490-503 